\title{
Wearable Wireless Calling System in Hospitals based on ZigBee
}

\author{
Sheng Li, Yongjie Yang ${ }^{*}$, and Junjiao Zhang \\ School of Electronics and Information, Nantong University, Nantong, 226019, China
}

\begin{abstract}
With the continuous optimization and reform of the healthcare system, many of the equipment in hospitals are developing in the direction of intelligence. At present, the hospital bed call system relies mainly on the traditional cable. Wired call system wiring is tedious and high in cost. It can sometimes cause short circuits, circuit breakages, and other problems, which will affect the timely treatment of patients. Based on ZigBee CC2530 software and hardware technology, this paper designs a wearable wireless calling system in hospitals based on ZigBee. ZigBee technology is a short distance wireless communication technology, which is characterized by low power consumption and low cost. This article sets up the style of the watch based on the ZigBee wireless communication hardware. The nurse can detect the patient's location at all times through the wireless call system device worn by the patient. When a patient is away from his or her hospital bed and suddenly needs care, the patient can call the nursing station according to the wireless communication system worn on his wrist. The nurse can arrive quickly and carry out the treatment, which can greatly alleviate any emergencies. The application of this wireless call system has significantly improved the level of hospital care and further promoted the development of hospital equipment in the direction of intelligence.
\end{abstract}

Keywords: ZigBee; wireless call system; CC2530; wearable; positioning

(Submitted on May 7, 2018; Revised on June 20, 2018; Accepted on July 25, 2018)

(C) 2018 Totem Publisher, Inc. All rights reserved.

\section{Introduction}

At present, communication between patients and nurses in hospitals is inconvenient, and there is a lack of an effective communication channel. We seek to develop a ZigBee-based call system for wrist watches in hospital wards [1]. This system can achieve heart rate detection, real-time location, replacement of drugs, and emergency help. Replacing drugs and emergency help involves sending the patient's information to the nursing station by pressing a specific key [2]. Nurses accurately locate the information sent by patients and then take effective measures based on this information, so that the patients can be treated in time. Currently, the hospital ward call system is fixed beside the hospital bed; it is not mobile nor convenient. This article focuses on the development of a single function portable wearable communication equipment, specifically for calling systems in hospitals. This equipment is simple in function, low in cost, and strong in practicality. It is more suitable for the use of certain groups of elderly patients. It can greatly improve the treatment of hospital patients and is thus an important need for the hospital. The innovation of this article is not to study new technology, but to combine existing mature and feasible technology that is not yet widely used in the medical field and create a practical, convenient product for hospitals.

\section{Overall Design of System}

\subsection{System Architecture}

The hospital wearable wireless calling system based on ZigBee is designed to make up for the shortcomings of the hospital cable ward and improve the communication efficiency between nurses and patients [3]. The wearable wireless calling system in hospitals is connected according to the star network. The transmission of information takes place through the Basic RF protocol. Each node in the Basic RF protocol has the same status. It does not need to be divided into coordinator,

\footnotetext{
* Corresponding author.

E-mail address: yang.yj@ntu.edu.cn
} 
router, or terminal. However, in order to distinguish the different functions of different nodes, we will divide the nodes into three categories according to the different functions of nodes [4]. The first node is connected to the computer client, which is the nursing station and is mainly used to receive information. We call it the receiving node. The second node is mainly installed in a fixed position in different wards. It is used to receive information that is then sent to the receiving node, and we call it the relay node. The third node is the node that is carried by the patient. When the patient goes to a different room or different area of the hospital, the node sends its own signal to the nearby relay node, and the relay node can receive the signal in time and send it to the receiving node. We call this node the sending node. From the receiving node to the relay node, and finally to the sending node, the star network connection is created, forming the complete ZigBee wireless network [5]. This is shown in Figure 1.

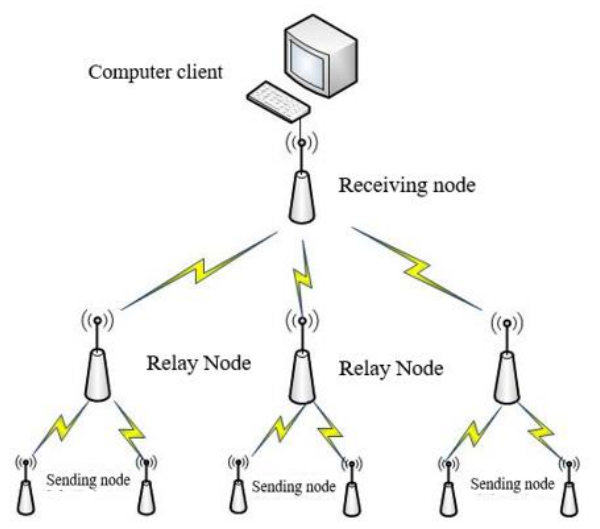

Figure 1. ZigBee wireless network connection

Patients sometimes walk around the hospital, such as to go to the bathroom. If they suddenly feel sick and need help, the patient can send an emergency rescue signal to the nursing station through the wireless wearable calling system he or she wears. At this point, the nearest relay node from the patient will receive the patient's information and store it. Then, it is sent to the receiving node along with the location information of the relay node itself. When the receiving node receives the signal, the nurse can collect the patient's personal information and current location in order to find and treat the patient. The schematic diagram of the communication frame of hospital wards is shown in Figure 2.

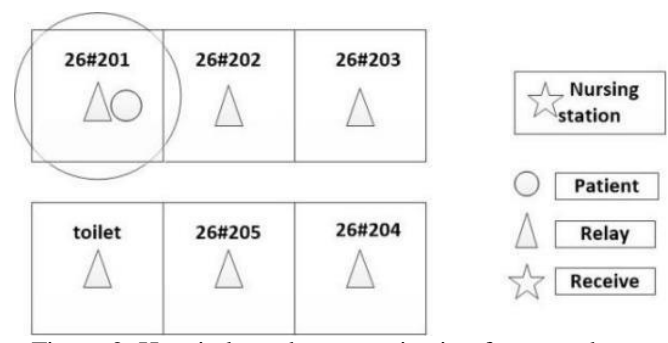

Figure 2. Hospital ward communication framework

\subsection{System Hardware Design}

The hardware of the wireless bed calling system is mainly composed of the main controller CC2530 module, power module, heart rate monitoring module, and key module [6]. The system hardware is shown in Figure 3.

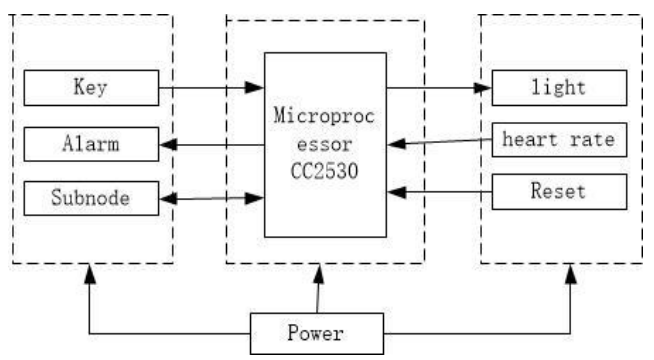

Figure 3. System hardware design 
The wireless communication module of the wearable wireless calling system in hospital is designed with CC2530 as the core chip [7]. The design of an antenna and balun matching circuit in the wireless sensor network based on ZigBee protocol is very necessary. It mainly relates to the excellent performance of RF channel indicators and the effect on communication distance and system power consumption. CC2530 can work normally by linking very few peripheral devices. The chip needs two crystal oscillators to provide clock operation for the chip: a $32 \mathrm{MHz}$ crystal oscillator and $32.768 \mathrm{KHz}$ crystal oscillator. The $32 \mathrm{MHz}$ crystal oscillator is mainly used to provide the frequency of sending and receiving RF transceivers [8]. The $32.768 \mathrm{KHz}$ crystal oscillator drives the sleep timer and watchdog timer. Because the wireless communication module works in the high-frequency band of $2.4 \mathrm{GHz}$, the components need to be rationally arranged in circuit design. The antenna layout requires the antenna to be as close as possible to the CC2530 chip and tries to keep it away from the external crystal oscillator as far as possible [9]. When transmitting data, the wireless communication module needs to divide the transmitted data into packets less than 128 bytes, which are stored in the transmit buffer. When making the transmitted data frame, the chip automatically adds the processed data to the preamble and begins to mark and check the code. Then, the digital signal is converted into an analog signal through a digital-analog conversion circuit and is transmitted from the antenna to the antenna under the filter's processing. Receiving data is the opposite of sending data. First, the high-frequency signals received are converted to low-frequency signals. Then, analog signals are converted to digital frames by digitalanalog circuits, the frame heads of the digital frames are stripped from the frame structure, and the data information is finally stored in the reception cache [10]. From wireless transmission to wireless reception, the basic communication of the wireless network is built. Figure 4 shows the circuit design of the CC2530 wireless communication module.

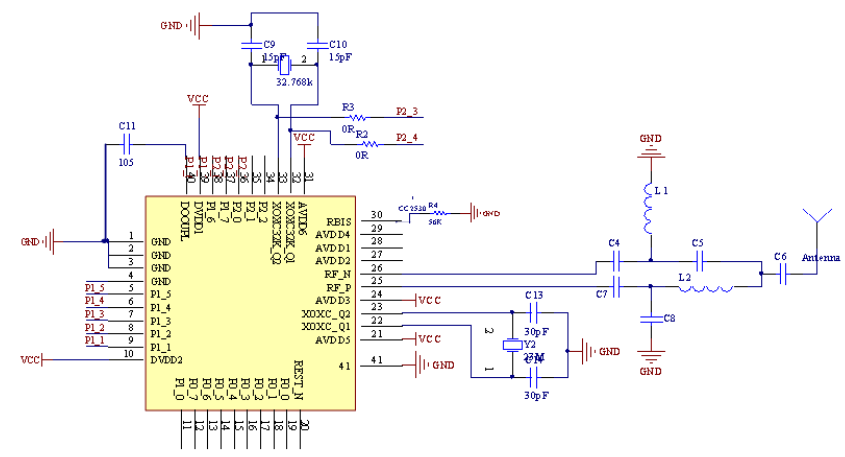

Figure 4. The circuit design of the CC2530 wireless communication module

\section{Software Design of System}

The software design of the system is mainly based on the establishment of a wireless network by ZigBee and is programmed using the IAR software development platform. The program design includes a system initialization design, launch node design, relay node design, and receiver node design. The receiving node is connected to the computer through the serial port. Then, by using the Visual Studio software to create a visual nursing station interface, the received digital information can be displayed through an edited nursing station interface [11].

\subsection{Design of Data Sending Software}

First, the user layer sends the destination address, the data buffer pointer, and the data buffer length to the Basic RF layer through the Basic RF function. A buffer is set up in the Basic RF layer to store the data that is ready to be sent. The data that is stuck in the Basic RF layer buffer is transferred to the CC2530 of the hardware layer to be sent to the FIFO buffer area, and the hardware layer is called to initiate ISTXON () to send the data out. The transmission channel will wait for the data to be sent out, obtains the sending result from the hardware layer, and sends the status to the Basic RF layer [12]. If the Basic RF layer receives the reply packets from the Basic RF sending function, it sends SUCCESS to the user level, indicating that the data is sent successfully. The process is shown in Figure 5.

\subsection{Design of Data Receiving Software}

First, when the hardware layer receives a packet, it will automatically apply for interruption, and the interrupt service function stops the Basic RF layer to read the data received by the hardware layer. The Basic RF layer will receive the first byte from the $\mathrm{CC} 2530$ in the buffer area, the receiving frame, and read all the data in the buffer layer of the hardware layer to the Basic RF layer. CC25320 automatically sends response signal ACK to the transmitter [13]. At the same time, the 
Basic RF layer will recognize the address of the received frame. If CRC is correct, it is received. The Basic RF layer will detect the FCS and serial number of the packet. If they are consistent with the expected signs, the new packets will be received and sent to the user level to send TRUE. The user layer will continue to query its logo through the received data. When the TRUE is returned, the user layer obtains the new data and RSSI values from the Basic RF layer and stores them in the memory, providing a pointer called pData for the data to be used by the user [14]. This process is shown in Figure 6.

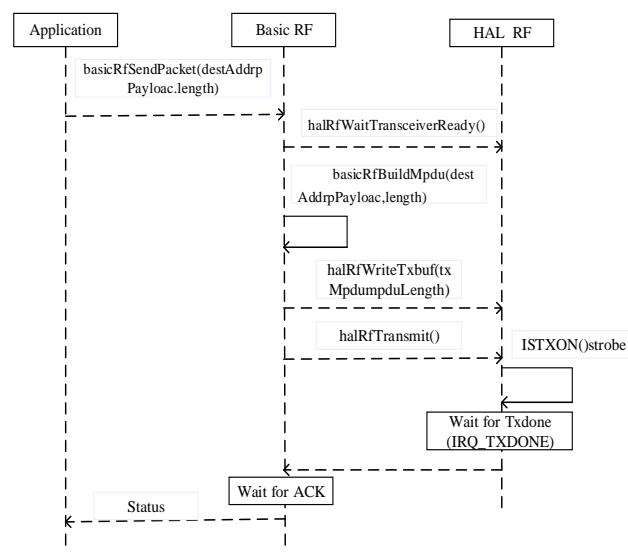

Figure 5. Design of data sending software

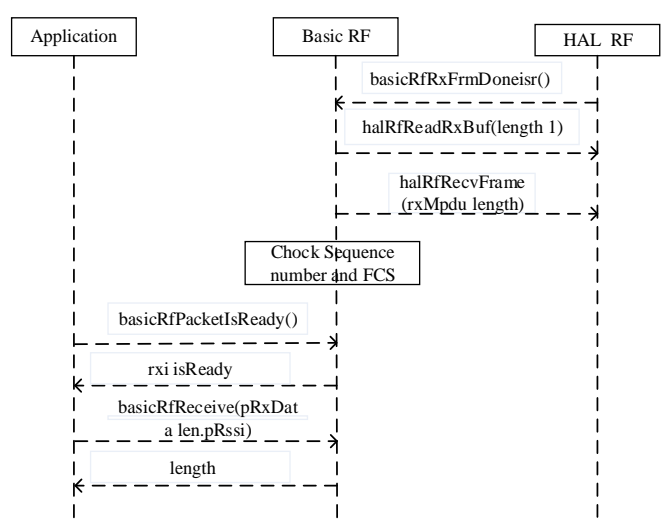

Figure 6. Design of data receiving software

\subsection{Software Design of Upper Computer}

The most important feature of creating Windows applications by using Visual Studio software is to provide various kinds of software for developers to use. It not only improves the speed of development, but also unifies the development interface. The Windows application is a based window application program, which is programmed by C\# and displayed on the user interface by Windows [15]. The interface mainly displays the patient's name, the location of the patient, the heart rate of the patient, and the current state of the patient. When a patient needs to change a drug or send an urgent distress signal, the patient sends the signal through a keystroke. At this time, the interface will show the corresponding information. The nurse who sees the message on the interface will take timely measures to carry out the targeted treatment. Figure 7 shows the overall process of nursing.

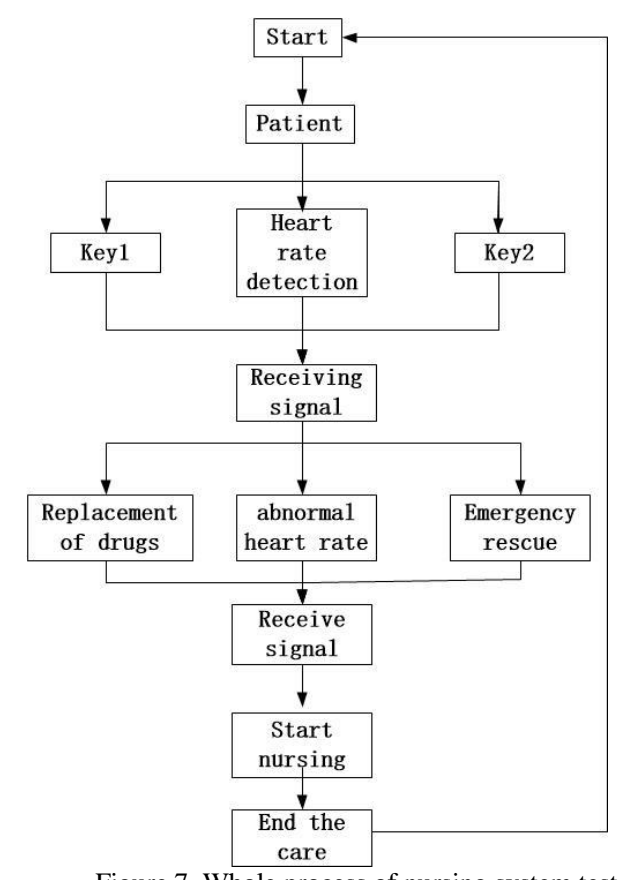

Figure 7. Whole process of nursing system test 
The system mainly receives data from the receiving node through the serial port. To enable users to read digital information in a popular way, we can visualize the numbers by writing the visual interface, as shown in Figure 8 . What you see at this time is the information of each patient. When the nurse receives the patient's call, the nurse can create an effective treatment using the information displayed on the interface. As shown in Figure 9, Wanger is showing an urgent distress signal. Xiaoming detects abnormal heartbeat information that needs to be processed [16]. At this time, nurses press the nursing button by clicking the visual interface. As shown in Figure 9, the state of Zhangsan is the state of nursing, so that targeted treatment can be achieved. This is the same for Xiaoming and Wanger. When the nurse completes the diagnosis and treatment of the patient, the nurse uses the interface to end the nursing, and then the user interface begins to run normally and converts to the normal interface of the patient in Figure 8.

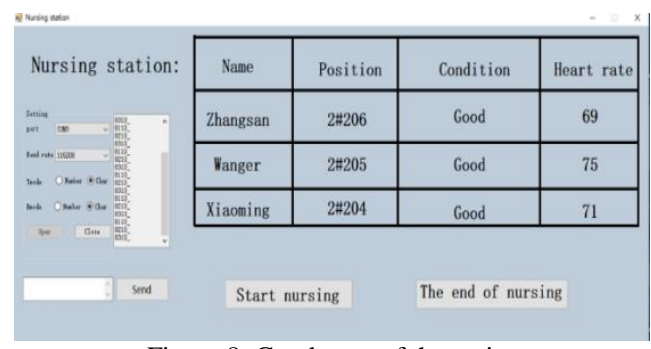

Figure 8. Good state of the patient

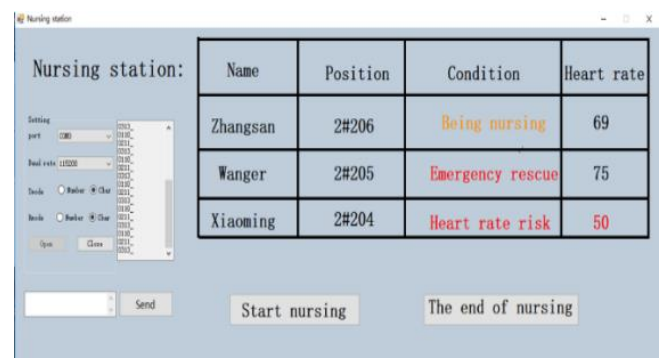

Figure 9. The patient is nursing and calling display

\section{Conclusions}

Through the design of a wearable wireless calling system in hospitals, we have achieved our desired effect through practice. According to the functional requirements of hospitals, it is believed that this system will become a necessity of hospitals in the near future. With our aging population and the big data era, this wearable medical and remote service mode, first introduced into hospitals, will eventually become the mobile terminal of the next era.

\section{Acknowledgements}

This work was supported by the Open Research Project of Nantong Intelligent Information Technology Research Center (KFKT2017B05) of Nantong University and the First Phase Project of Jiangsu University Brand Specialty Construction Project (PPZY2015B135). In addition, it was completed under the support of the Innovative Training Program for College Students in Jiangsu (201710304051Z). The authors thank the three anonymous reviewers for their helpful suggestions.

\section{References}

1. X. H. Cao, D. M. Shila, Y. Cheng, Z. Q. Yang, Y. Zhou, and J. M. Chen, "Ghost-in-ZigBee: Energy Depletion Attack on ZigBee-based Wireless Networks,” IEEE Internet of Things Journal, Vol. 3, No. 5, pp. 816-829, 2016

2. M. Li and Y. T. Kim, "Development of Patch-Type Sensor Module for Wireless Monitoring of Heart Rate and Movement Index," Sensors \& Actuators A Physical, Vol. 173, No. 1, pp. 277-283, 2012

3. T. Kim and D. Kim, "Opportunistic Shortcut Tree Routing in ZigBee Networks," IEEE Sensors Journal, Vol. 16, No. 12, pp. 5107-5115, 2016

4. S. Kumar, V. Pavithra, R. Banu, et al. "Smart Home Energy Management System including Renewable Energy based on Zigbee and ARM9 Microcontroller," Social Science Electronic Publishing, 2017

5. A. Kumar and G. P. Hancke "A Zigbee-based Animal Health Monitoring System,” IEEE Sensors Journal, Vol. 15, No. 1, pp. 1, 2015

6. F. Shariff, N. A. Rahim, and W. P. Hew, "Zigbee-based Data Acquisition System for Online Monitoring of Grid-Connected Photovoltaic system," Pergamon Press, Inc. 2015 
7. C. Peng, K. Qian, and C. Wang, "Design and Application of A VOC-Monitoring System based on A ZigBee Wireless Sensor Network," IEEE Sensors Journal, Vol. 15, No. 4, pp. 2255-2268, 2015

8. L. Luoh, "ZigBee-based Intelligent Indoor Positioning System Soft Computing," Springer-Verlag, 2014

9. S. Choudhury, P. Kuchhal, R. Singh, and Anita, "ZigBee and Bluetooth Network based Sensory Data Acquisition System 诂," Procedia Computer Science, Vol. 48, pp. 367-372, 2015

10. Z. Q. Wang and Z. H.Huang, "Wearable Health Status Monitoring Device for Electricity Workers using ZigBee-based Wireless Sensor Network," in Proceedings of International Conference on Biomedical Engineering and Informatics, pp. 602-606, 2015

11. S. Amann, S. Proksch, S. Nadi, and M. Mezini, "A Study of Visual Studio Usage in Practice," in Proceedings of IEEE International Conference on Software Analysis, Evolution, and Reengineering, pp. 124-134, 2016

12. L. Luoh, "ZigBee-based Intelligent Indoor Positioning System Soft Computing," Soft Computing, Vol. 18, No. 3, pp. 443-456, 2014

13. F. Cuomo, S. D. Luna, U. Monaco, and F. Melodia, "Routing in ZigBee: Benefits from Exploiting the IEEE 802.15 .4 Association Tree," pp. 3271-3276, 2015

14. H. Y. Tung, K. F. Tsang, K. T. Chui, H. C. Tung, H. R. Chi, G. P. Hancke, and K. F. Man, "The Generic Design of A HighTraffic Advanced Metering Infrastructure using ZigBee," IEEE Transactions on Industrial Informatics, Vol. 10, No. 1, pp. 836844, 2014

15. P. Ritchie, "Introduction to Visual Studio 2015," Apress, 2016

16. M. Li and Y. T. Kim, "Development of Patch-Type Sensor Module for Wireless Monitoring of Heart Rate and Movement Index," Sensors \& Actuators A Physical, Vol. 173, No. 1, pp. 277-283, 2012

Sheng $\mathbf{L i}$ is currently a graduate student at the School of Electronic Information at Nantong University. His main research interests include the Internet of things and embedded systems.

Yongjie Yang is a graduate of Nanjing University of Science and Technology and the dean of the School of Electronic Information at Nantong University. He has participated in national and provincial studies, and his main research direction is in wireless communication.

Junjiao Zhang is currently a graduate student at the School of Electronic Information at Nantong University. Her main research interests include the Internet of things and embedded systems. 